\title{
Fundamental Insights in Photoelectrocatalytic Hydrogen Production with a Hole-transport Bismuth Metal-organic Framework
}

\author{
Alba García-Sánchez ${ }^{\mathrm{a}}$, Miguel Gomez-Mendoza ${ }^{\mathrm{a}}$, Mariam Barawi ${ }^{\mathrm{a}}$, Ignacio J. Villar-Garcia ${ }^{\mathrm{a}}$, Marta Li- \\ $\operatorname{ras}^{\mathrm{a}}$, Felipe Gándara ${ }^{\mathrm{b} *}$, Víctor A. de la Peña O'Shea ${ }^{\mathrm{a} *}$

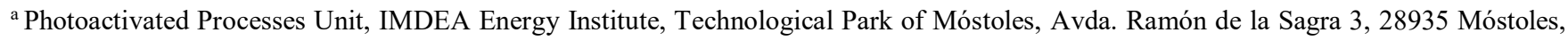 \\ Madrid, Spain. \\ ${ }^{\mathrm{b}}$ Instituto de Ciencia de Materiales de Madrid, CSIC, Sor Juana Inés de la Cruz 3, 28049 Madrid, Spain
}

KEYWORDS. Metal-organic framework, photoelectrochemistry, charge transfer, hydrogen production.

\begin{abstract}
Solar fuels production is a cornerstone in the development of emerging sustainable energy conversion and storage technologies. Light-induced $\mathrm{H}_{2}$ production from water represents one of the most crucial challenges to produce renewable fuel. Metalorganic frameworks (MOFs) are being investigated in this process, due to the ability to assemble new structures with the use of suitable photoactive building blocks. However, the identification of the reaction intermediates remains elusive, having negative impacts in the design of more efficient materials. Here, we report the synthesis and characterization of a new MOF prepared with the use of bismuth and dithieno[3,2-b:2',3'-d] thiophene-2,6-dicarboxylic acid (DTTDC), an electron-rich linker with hole transport ability. By combining theoretical studies and time-resolved spectroscopies, such as core hole clock and laser flash photolysis measurements, we have completed a comprehensive study at different time scales (fs to-ms) to determine the effect of competitive reactions on the overall $\mathrm{H}_{2}$ production. We detect the formation of an intermediate radical anion upon reaction of photogenerated holes with an electron donor, which plays a key role in the photoelectrocatalytic processes. These results shed new light on the use of MOFs for solar fuel production.
\end{abstract}

\section{INTRODUCTION}

In the search for carbon neutral technologies able to provide sustainable energy, it is crucial to develop processes that effectively exploit renewable natural resources. In this context, scientists are allocating efforts to convert and store solar energy into chemicals and fuels, mimicking photosynthesis. ${ }^{1,2,3,4}$ Currently, the development of artificial photosynthesis depends on the search for new, efficient photocatalytic materials able to be used as photoelectrodes in photoelectrochemical cells (PEC) to produce solar fuels such as water splitting ${ }^{5}$, or $\mathrm{CO}_{2}$ photoreduction. ${ }^{6}$ Generally speaking, a photoelectrocatalyst is a material that can be activated upon light absorption, leading to charge generation and separation of electron-hole $\left(\mathrm{e}^{-}-\mathrm{h}^{+}\right)$pairs that trigger both reduction and oxidation reactions. Metal oxides are the most commonly used in this research topic. However, they suffer of several limitations such as high recombination rates of charge carriers, low charge separation efficiency, slow water oxidation kinetics, as well as limited absorption across the light spectrum, generally leading to limited efficiencies. To overcome these problems, different strategies are being followed including the use of sensitizers, co-catalysts, dopants, band-gap engineering, or heterojunctions, with the ultimate goal of improving their activity as photoelectrodes in photoelectrocatalytic systems. ${ }^{7,8,9}$

Metal-organic frameworks (MOFs) are porous crystalline materials built by the combination of metal ions and clusters with organic linkers. MOFs are emerging in the field of photocatalysis and photoelectrochemistry ${ }^{10,11,9}$ due to their tailorable capacity to absorb light by the judicious selection of metal cluster and organic linkers. ${ }^{10}$ This ability has been described in a number of reports available in the literature concerning the use of MOFs as photocatalysts. ${ }^{12,13,14,15}$ However, their development for photoelectrocatalyic processes is still scarce and most published works are focused on MOFs as visible photosensitizers in combination with metal oxides, ${ }^{16,17,18,19,20}$ or metal oxide structures obtained from MOF calcination. ${ }^{21,22,23}$ Only few examples are reported using bare MOFs, probably due to their limited stability in water against photocorrosion processes. Furthermore, in most examples, $\mathrm{H}_{2}$ quantification is not included and only density currents are measured. In a recent work, Padial et al. ${ }^{24}$ studied the photoelectrochemical properties of a hydroxamate Ti-MOF with siderophore-type linkers using $\mathrm{Na}_{2} \mathrm{SO}_{3}$ as electron donor, which exhibits a $\mathrm{H}_{2}$ production of $4.5 \mu \mathrm{mol} / \mathrm{cm}^{2}$ in one hour. Ifraemov et al. ${ }^{25}$ have demonstrated that a MOFbased electrode can be switched from photo-anodic to photocathodic, and tested it as photocathode in PEC, reaching 0.112 $\mu \mathrm{mol} \mathrm{H}_{2}$ after $7 \mathrm{~h}$ (at $-0.8 \mathrm{~V}$ vs. NHE).

It is also worthy to note that none bismuth based porous MOF has been reported thus far for $\mathrm{PEC} \mathrm{H}_{2}$ production. Most importantly, the role of photogenerated charges in the mechanisms involving the formation of active intermediates and products using MOFs still remains unknown. This is mainly due to the different time scales in which the multielectronic processes take place, which hinders the unambiguous identification of the involved species. ${ }^{6}$

In the present work, we report the synthesis and characterization of a new bismuth based MOF, denoted IEF-5, (IMDEA 
Energy Framework-5), specifically prepared for its use as a photoelectrode for hydrogen evolution reaction by the choice of an electron rich linker with hole-transport ability. Furthermore, we have completed a series of theoretical and time-resolved spectroscopic studies at different time scales, from fs to ms, to unravel the effects of photoinduced charge dynamics in the photoelectrocatalytic hydrogen production mechanism. The understanding of the overall process provides a unique insight for the development of photoelectroactive materials for light induced reactions.

\section{EXPERIMENTAL SECTION}

X-Ray diffraction. Single-crystal data were obtained in a Bruker four-circle kappa diffractometer equipped with a $\mathrm{Cu}$ INCOATED microsource $(\mathrm{Cu} \mathrm{K} \alpha$ radiation, $\lambda=1.54178 \AA$ ), and a Bruker PHOTON I area detector (CPAD Technology). Samples were also characterized by X-ray powder diffraction (XRD) using a Philips PW 3040/00 X'Pert MPD/MRD diffractometer with $\mathrm{Cu} \mathrm{Ka}$ radiation $(\lambda=1.54178 \AA)$ at a scanning rate of $0.2^{\circ} \mathrm{s}^{-1}$ from 3 to $40^{\circ}$.

X-Ray photoelectron spectroscopy (XPS). XP spectra of the powder sample IEF-5 deposited on carbon based double side sticky tape were recorded were recorded using a SPECS NAPXPS System incorporating the DeviSim NAP reaction cell. The spectrometer was equipped with an $\mathrm{Al} \mathrm{K} \alpha$ monochromated source $(\mathrm{h} v=1486.6 \mathrm{eV})$, composed of a SPECS XR50 MF xray gun and a $\mu$-FOCUS 600 monochromator, and PHOIBOS 150 NAP 1D-DLD analyzer. X-ray gun power was set to $25 \mathrm{~W}$ (1.68 mA emission current and $15 \mathrm{kV})$. With this X-ray settings, the intensity of the $\mathrm{Ag} 3 \mathrm{~d}_{5 / 2}$ photoemission peak for a $\mathrm{Ag}$ sample, recorded at $10 \mathrm{eV}$ pass energy $(\mathrm{PE})$, was $1 \times 10^{4} \mathrm{cps}$ and the full width at half maximum (FWHM) was $0.60 \mathrm{eV}$. Binding energy calibration was made using $\mathrm{Au} 4 \mathrm{f}_{7 / 2}(84.01 \mathrm{eV}), \mathrm{Ag} 3 \mathrm{~d}_{5 / 2}$ $(368.20 \mathrm{eV})$ and $\mathrm{Cu} 2 \mathrm{p}_{3 / 2}(932.55 \mathrm{eV})$. The survey scans were acquired using $30 \mathrm{eV}$ pass energy, $1 \mathrm{eV}$ step size and 7 seconds (1 s x 7 scans) dwell times. O 1s, C 1s, Bi 4f and S 2p high resolution spectra were acquired using $10 \mathrm{eV}$ pass energy, 0.1 eV step size and 10 seconds (1s x 10 scans) dwell times. The sample was analyzed at an electron take-off angle normal to the surface with respect to the analyzer. The analyzer is set up at an angle close to the magic angle of $54.7^{\circ}$ with respect to the x-ray beam.

Core Hole Clock (CHC) measurements. X-ray absorption (XAS) and Auger decay measurements on drop casted IEF-5 films were performed at the B07-1 Ambient Pressure branchline at VERSOX (Diamond Synchrotron). The source is a bending magnet resulting on a photon flux $>1 \times 10^{10} \mathrm{ph} / \mathrm{s}$ and the hemispherical electron energy analyzer is a Phoibos NAP150 from SPECS. XAS spectra were recorded using $0.3 \mathrm{eV}$ step and 10 seconds dwell time with Auger snapshots at PE200 eV. Auger spectra (for $\mathrm{CHC}$ experiments) were collected using $10 \mathrm{eV}$ pass energy and $0.1 \mathrm{eV}$ step size at different dwell times depending on signal to noise ratios. No beam damage effects were observed in the photoabsorption and photoemission spectra over-time. Casa XPS was used for data interpretation. Shirley or two-point linear background subtractions were employed depending on background shape. Peaks were fitted using GL(30) line-shapes; a combination of a Gaussian (70\%) and Lorentzian $(30 \%)$.
Laser Flash Photolysis (LFP). The laser flash photolysis equipment is based on a pump-probe setup purchased from Edinburgh Co (LP980-K). The pump source is an optical parametric oscillator (OPO) pumped by the third harmonic of a Nd:YAG laser (EKSPLA). The excitation wavelength employed for the measurements was of $355 \mathrm{~nm}$. The single pulses were $c a .5 \mathrm{~ns}$ duration, and the energy lower than $1 \mathrm{~mJ} /$ pulse. A pulsed xenon flash lamp $(150 \mathrm{~W})$ was employed as detecting light source. A monochromator (TMS302-A, grating 150 lines $/ \mathrm{mm}$ ) disperses the probe light after it has passed the sample. The probe light is then passed on to a PMT detector (Hamamatsu Photonics) to obtain the temporal resolved picture. The absorbance of the solutions was kept at 0.3 at $\lambda_{\text {exc }}=355$ $\mathrm{nm}$ both for the free ligand and the corresponding IEF-5 (as dispersed solution). All transient spectra were recorded at room temperature using $10 \times 10 \mathrm{~mm}^{2}$ quartz cells with $3 \mathrm{~mL}$ capacity and were bubbled for 15 min with $\mathrm{N}_{2}$ before acquisition. Triplet lifetimes of dyads were measured in aqueous solution, from the monoexponential fitting of the decay traces registered at 440 and $520 \mathrm{~nm}$.

Quenching Experiments. In a typical quenching experiment, the appropriate volumes of a freshly prepared $\mathrm{Na}_{2} \mathrm{SO}_{3}$ solution (2 M) as electron donor, were added to the aerated or purged DTTDC solution or IEF-5 suspensions. The absorbance of the samples was kept at 0.1 or 0.3 at $\lambda_{\mathrm{exc}}=355 \mathrm{~nm}$ for fluorescence or LFP experiments, respectively. In analogous experiments, chloroplatinic acid $\left(\mathrm{H}_{2} \mathrm{PtCl}_{6}\right)(0.25 \% \mathrm{wt})$ was employed as electron acceptor. All the photophysical measurements were performed at room temperature in a quartz cell of $1.0 \mathrm{~cm}$ optical path length.

Photoelectrochemical measurements. Experiments were performed in a three-electrode glass cell with a quartz window containing an aqueous solution of $0.5 \mathrm{M} \mathrm{Na}_{2} \mathrm{SO}_{3}$ for photoelectrochemical measurements and $0.1 \mathrm{M}$ of $\left.\left[\left({ }^{\mathrm{n}} \mathrm{Bu}\right)_{4}\right) \mathrm{N}\right] \mathrm{PF}_{6}$ in acetonitrile for electrochemical characterization. IEF-5 powders were dispersed in ethanol and then deposited by drop casting on ITO cover glasses and used as working electrodes. The counter electrode was a platinum wire, and the reference one was an $\mathrm{Ag} / \mathrm{AgCl}$ electrode. Voltage and current density (at dark and under illumination) were measured with a potentiostat-galvanostat PGSTAT204 provided with an integrated impedance module FRAII $(10 \mathrm{mV}$ of modulation amplitude is used at 400Hz). A solar Simulator (LOT LSH302 Xe lamp and a LSZ389 AM1.5 Global filter) was used as a light source.

Computational details. Theoretical calculations by periodic density functional theory (DFT) were carried out using crystallographic data of IEF-5. Geometry and electronic structure were performed using the projected augmented wave method implemented in Viena ab initio simulation package (VASP). ${ }^{26,27}$ The total energies corresponding to the optimized geometries of all samples were calculated using the spin polarized version of the Perdew-Burke-Ernzerhof (PBE) ${ }^{28}$ and the Heyd-Scuseria-Enzerhof hybrid functional (HSE06). ${ }^{29}$ The cut-off for the kinetic energy of the plane-waves was set to $450 \mathrm{eV}$ to ensure a total energy convergence better than $10^{-4} \mathrm{eV}$. The VESTA package v.3.3.9 ${ }^{30}$ was used to represent the electron localization. Time dependent Density Functional Theory (TD-DFT) ${ }^{31,32}$ implemented in the software Gaussian $16^{33}$ employing a B3LYP functional. ${ }^{34,35,36}$ The standard 6-31G(d.p) and 
LAND2DZ $Z^{37,38,39}$ basis was chosen for ligand and IEF-5 respectively. In the case of TD-DFT calculations for Bi-MOF a fix number of 400 states were selected.

\section{Results and Discussion}

MOF synthesis and characterization. Our strategy to minimize charge recombination while allowing visible light absorption is based on the selection of a linker based on fused heterocycles. Condensed thiophene $\pi$-systems have been successfully used as building blocks for materials with electronic and optical properties. Dithieno[3,2-b:2',3'-d] thiophene (DTT) comprises three fused thiophene rings, which create an electron rich rigid core that alongside $\pi$-staking permit electronic delocalization and mobility. This moiety has been used in photo- and electroluminescent devices, two-photon absorption and excited fluorescence, non-linear optical chromophores and photocromic materials. ${ }^{40}$ In particular, the 2,6-dicarboxylic acid functionalized DTT, dithieno[3,2-b:2',3'-d] thiophene-2,6-dicarboxylic acid (DTTDC, Scheme 1, S1 and S2), appears as a suitable linker to reticulate fused thiophene units into MOF crystals. ${ }^{41,42,43}$

Scheme 1: Dithieno[3,2-b:2',3'-d]thiophene-2,6-dicarboxylic acid (DTTDC) is reacted with bismuth nitrate to form IEF-5.

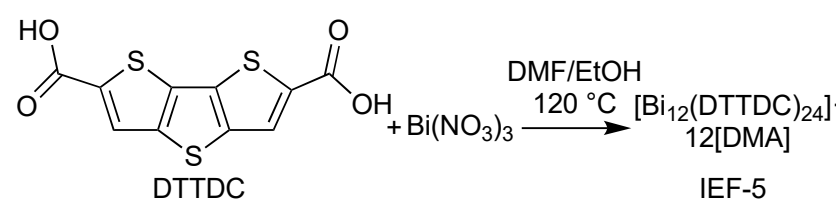

The solvothermal reaction between DTTDC and $\mathrm{Bi}\left(\mathrm{NO}_{3}\right)_{3} \cdot 5 \mathrm{H}_{2} \mathrm{O}$ in a mixture of DMF and ethanol at $120{ }^{\circ} \mathrm{C}$ for $72 \mathrm{~h}$ resulted in the formation of IEF-5 in the form of orange crystals (see supporting information, $\mathbf{S 1}$, for full experimental details). Single crystal X-ray diffraction (SCXRD) analysis revealed that IEF-5 crystallizes in the monoclinic system, space group $C 2$ with lattice parameters $\mathrm{a}=25.5961(19), \mathrm{b}=$ $22.5938(19), c=17.2880(2), \beta=100.512(8)^{\circ}$. Table $\mathbf{S 1}$ contains full crystallographic and refinement details. The framework of IEF-5 is formed by an inorganic secondary building unit (SBU) consisting of three bismuth atoms linearly arranged, coordinated to twelve carboxylic groups from the organic linkers (Figure 1a). The Bi-O distances range between 2.299(14) and 3.078(8) A. Each inorganic SBU is connected to six neighboring ones with pairs of parallel linkers, resulting in the formation of a pcu network (Figure 1b). There are two interpenetrated networks in the structure of IEF-5, with presence of DMF and water molecules located between them. Considering the SBU formula of $\left[\mathrm{Bi}_{3}\left(-\mathrm{CO}_{2}\right)_{12}\right]^{3-}$, the presence of cations is required for balancing the framework charge. Despite, the location of the counterions could not be unambiguously determined from the SCXRD refinement, the results of the elemental analysis and XPS are consistent with the presence of twelve dimethyl ammonium cations per unit cell, which are formed by decomposition of DMF solvent molecules, and resulting in a final MOF formula of $\left[\mathrm{Bi}_{12}(\mathrm{DTTDC})_{24}\right] \cdot 12[\mathrm{DMA}] \cdot 4 \mathrm{DMF} \cdot 2 \mathrm{H}_{2} \mathrm{O}$ $(32.61 \% \mathrm{C} ; 2.20 \% \mathrm{~N} ; 1.74 \% \mathrm{H} ; 22.67 \% \mathrm{~S}$ calc. and $31.72 \% \mathrm{C}$; $2.160 \% \mathrm{~N} ; 1.35 \% \mathrm{H} ; 21.97 \% \mathrm{~S}$ found). A plausible location of the DMA cations was determined with DFT calculations (Figure 1c). The phase purity of IEF-5 was determined by comparison of the powder X-ray diffraction (PXRD) pattern with the one calculated from the single crystal structure (Figure S1). During the optimization of the synthetic procedure, another MOF crystal was identified (IEF-6), which according to the SCXRD analysis consists of a monometallic SBU, formed by a bismuth cation coordinated to four DTTDC linkers, resulting in stacked square layers. However, it has not been possible to isolate this MOF as a pure phase yet. Section S2 contains structural details of this material (Table S2 and Figure S2).

a
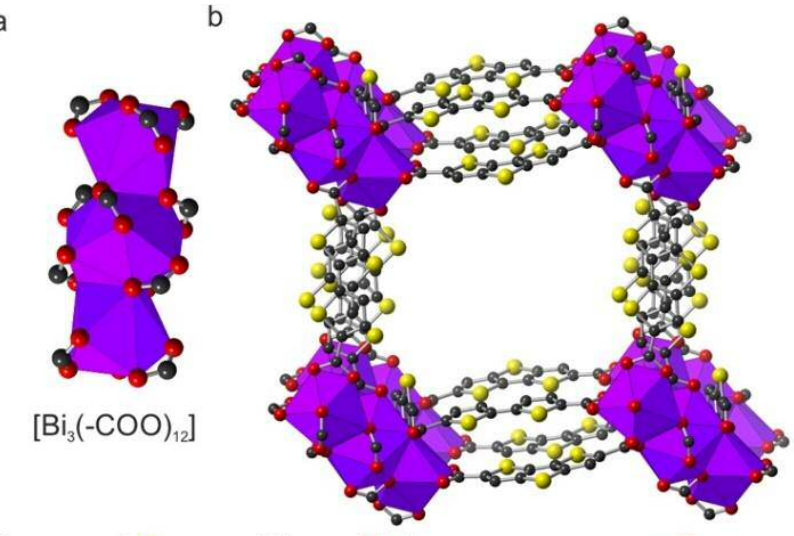

C

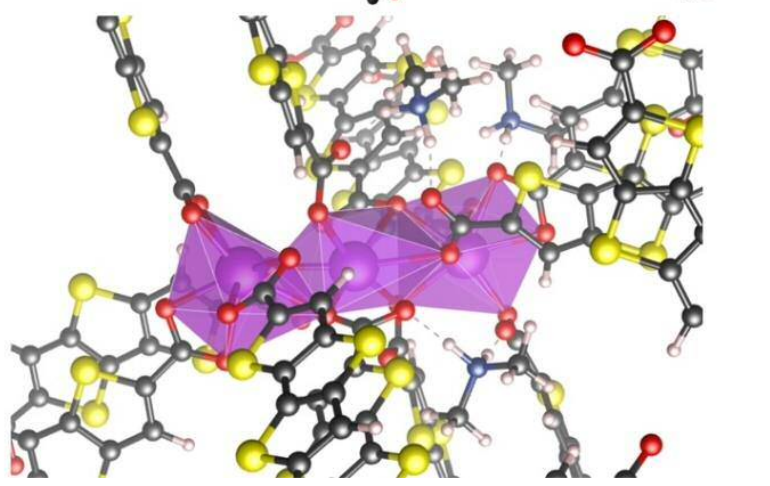

Figure 1. a) The inorganic SBU in IEF-5 is formed by three bismuth atoms. b) Each SBUs is connected to other six ones, forming a pcu type network. c) Representation of the simulated interaction of the DMA cations with the framework atoms. Bismuth is purple, oxygen is red, carbon is black, sulphur is yellow and nitrogen is blue.

Additional spectroscopic characterization including FTIR (Figure S3, and Table S3 and S4) and solid state NMR (Figure S4) further confirms the purity of the IEF-5 sample. XPS spectra complete the chemical information and electronics environments (Figure S5). The N1s signal is characteristic of DMF molecules encapsulated in crystalline networks (Figure S6). The Bi 4f signal (Figure $\mathbf{S 7}$ a) is characteristic of $\mathrm{Bi}^{3+}$ atoms (160.0 eV). The C1s signal (Figure S7 b) can be deconvoluted with three main contributions at $285.2 \mathrm{eV}$ (C-C bonds), 286.3 (C-S bonds) and $288.7(\mathrm{O}=\mathrm{C}-\mathrm{C})$ and the $\mathrm{S} 2 \mathrm{p}$ signal of thiophene containing polymers (Figure S7 b). ${ }^{44}$ The calculated percentages (Table S5) match the theoretically calculated ones within error. Valence band (VB) region of XPS also permits to determine the Fermi edge (Figure S8). IEF-5 exhibits a surface area of $30 \mathrm{~m}^{2} \mathrm{~g}^{-1}$ calculated by BET (Brunauer-Emmett-Teller) theory, according to the $\mathrm{N}_{2}$ adsorption/desorption isotherm recorded at $77 \mathrm{~K}$ (Figure S9). The low surface area measured is consistent with presence of DMA cations in the pores. Structural stability experiments were performed by thermogravimetric analysis, which shows no significant weight loss below 250 
${ }^{\circ} \mathrm{C}$, both in argon and air streams (Figure S10). Beyond this temperature, the material begins to decompose, as proved by the $50 \%$ weight loss observed in the curve recorded in argon atmosphere ( $70 \%$ in air). Thermodiffraction experiments show that the structure is thermally stable up to $c a .220^{\circ} \mathrm{C}$, with no significant changes in the PXRD patterns (Figure S11). Chemical stability of IEF-5 was tested by soaking the MOF in common solvents (Figure S12). IEF-5 stability was also tested in sodium sulfite solution (Figure S13-S17), which was used as the electrolyte in the photoelectrocatalytic measurements.

Optoelectronic characterization. Upon successful synthesis of a novel MOF incorporating the hole transport DTTDC linker, we turned our efforts to fully understand the light induced charge transfer properties and their influence on the photo(electro)catalytic activity. For this purpose, we have combined electrochemical and time resolved optical techniques with theoretical calculations to thoroughly characterize the optoelectronic features of the linker and IEF-5. UV-vis absorption spectrum of DTTDC shows a band with an edge at $375 \mathrm{~nm}$ and a maximum at $335 \mathrm{~nm}$. These transitions are related to HOMO and HOMO1 to LUMO orbitals (see Figure S18 and Table S6).

DFT calculations were also performed to determine the electronic structure of IEF-5 and its effect on the photo(electro)catalytic behavior. Figure 2a shows the density of states (DOS) of IEF-5 where valence band (VB) is composed of C2p, O2p and $\mathrm{S} 3 \mathrm{p}$ orbitals, while conduction band $(\mathrm{CB})$ is comprised mainly by the linker $\mathrm{C} 2 \mathrm{p}$ and $\mathrm{S} 2 \mathrm{p}$ states, while the $\mathrm{Bi} 2 \mathrm{~s}$ contribution is significantly smaller and at higher energies (ca. $3.5 \mathrm{eV}$ ). The energy of the fermi level is $\mathrm{E}_{\mathrm{F}}=0.95 \mathrm{eV}$, in near agreement with the value obtained by XPS ( $E_{F}=1.1 \mathrm{eV}$, see Figure S8). In addition, electron localization function (ELF) plots (Figure 2b) show an evident charge distribution mainly in the aromatic rings and in the sulphur atoms. Related electronic distribution where conduction band contributions are located in the ligand and metal, which facilitate the ligand to ligand charge transfer, have been previously reported. ${ }^{24}$ To confirm this fact, we also perform TD-DFT calculations with a cluster of this MOF (see Figure 2c and Section S4). The results exhibit a high number of electronic transitions mainly attributed to vibronic states, which corroborate the higher contribution of ligand to ligand charge transfer (Figure S19).

Theoretical studies show a band gap of $\mathrm{E}_{\mathrm{g}}=2.2 \mathrm{eV}$, which agrees with the optical measurements determined by UV-Vis diffuse reflected spectroscopy, using the Tauc method as an indirect transition (Figure S20). Cyclic voltammetry measurements (Figure 2d) indicate an electronic band gap energy of $2.4 \pm 0.1 \mathrm{eV}$, in line with the measured optical band gap (usually the optical and electrochemical band gaps differ between 0.2 or $0.3 \mathrm{eV}){ }^{24}$ The combination of these theoretical and experimental studies makes possible to determine the band energy diagram of IEF-5.

Photoelectrochemical measurements (PEC). These studies were carried out with the IEF-5 thin film electrodes over indium tin oxide (ITO). These films show a mean height of $2.5 \mu \mathrm{m}$ determined by atomic force microscopy (Figure 2e, Figure S21 and Table S7). The measurements were performed in a threeelectrode glass cell, using a solar simulator (A.M. 1.5) (Figure S22). The observed signals, along with the positive value of the photopotential measurements (Figure 2f) are indicative of a certain p-type semiconductor behavior of IEF-5. This Bi-MOF presents both positive and negative photoresponses in the linear sweep voltammetry. This can be explained by the position of the fermi level, determined by the flat band potential, which is not so close to the valence band. The energy of the flat band potential was determined as the point in which positive photocurrents changes to negative ones, as at this potential there is no band bending (Figure 2f).

The highest photocurrent values are observed at positive potentials, supporting its use as a photoanode. This observed photoconductivity is likely due to ligand-ligand charge transfer involving sulphur-sulphur interactions. In a related example, Park et al. found that isostructural MOFs $\mathrm{M}_{2}$ (TTFTB) $(\mathrm{M}=\mathrm{Mn}, \mathrm{Co}$, $\mathrm{Zn}$ and $\mathrm{Cd} ; \mathrm{H}_{4}$ TTFTB $=$ tetrathiafulvalene tetrabenzoate) exhibited a striking correlation between their single-crystal conductivities and the shortest $\mathrm{S} \cdots \mathrm{S}$ interaction defined by neighboring tetrathiafulvalene (TTF) cores, which inversely correlated with the ionic radius of the metal ions. ${ }^{23}$ This behavior may be enhanced in the case of IEF-5 favoring photoconductivity, due to the $\pi-\pi$ staking of the DTTDC ligand.

IEF-5 shows a positive photovoltage of $0.04 \mathrm{~V}$ vs $\mathrm{Ag} / \mathrm{AgCl}$ (Figure S23). Moreover, changes in the immediate signal in photocurrent measurements of IEF-5 with chopped illumination demonstrate the photoactivity of the MOF (Figure 2f) along different bias potentials. In the case of chronoamperometric measurements (Figure 2g), the cell was illuminated with on/off cycles with regular intervals. IEF-5 shows a stable behavior, with photogenerated current densities of $c a .6 .5 \mu \mathrm{A} \cdot \mathrm{cm}^{-2}$ at $0.3 \mathrm{~V}$. Moreover, we perform chronoamperometries at different light illumination intensities in order to investigate the possible existence of recombination losses during the photo-anodic reaction (Figure S24). Results confirms a linear behavior of the photocurrents with the illumination intensity, demonstrating that the recombination losses do not govern the charge transfer process. 



e
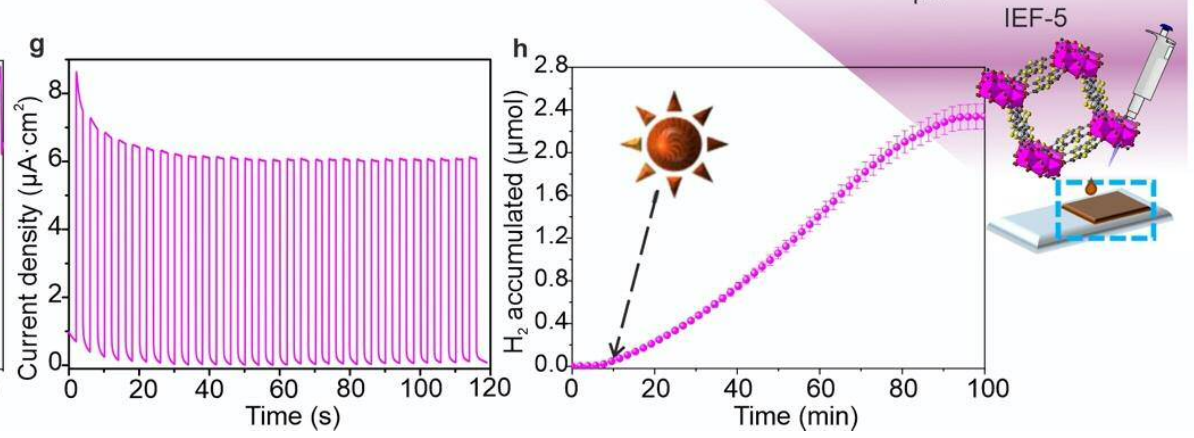

Figure 2 a) Atom-projected partial density of states (PDOS) for Bi2s (magenta), Bi2p (blue), C2p (grey), sulphur (yellow), oxygen (red), b) ELF isosurfaces and sections for IEF-5, atom color: Bi (magenta), C (brown), H (white) ,S (yellow), O (red); c) Solid UV-Vis spectra of IEF-5, TD-DFT (6-31G**) calculated excitation energies (vertical bars) and selected molecular orbitals, d) cyclic voltammetry measurement of IEF-5 in non-aqueous electrolyte at a scan rate of $50 \mathrm{mVs} /$, and experimental band diagram obtained with the water splitting redox couples at $\mathrm{pH}=0$, e) Scheme of MOF electrodes films deposited onto ITO cover glasses and AFM topography image; f) Linear sweep voltammetry under chopped illumination showing the photocurrent density at different bias potentials; g) Chronoamperometry at $0.3 \mathrm{~V}$ bias potential under chopped illumination; h) Photoelectrochemical $\mathrm{H}_{2}$ production accumulated in 60 min under AM1.5 irradiation at $0.35 \mathrm{~V}$.

Following, we performed photoelectrocatalytic $\mathrm{H}_{2}$ evolution reaction (HER) experiments under simulated solar irradiation (A.M. 1.5) and $0.35 \mathrm{~V}$ vs $\mathrm{Ag} / \mathrm{AgCl}$ applied potential. A $0.5 \mathrm{M}$ $\mathrm{Na}_{2} \mathrm{SO}_{3}$ solution was employed as electrolyte and hole scavenger. The PEC cell was connected to a gas chromatograph and an argon flow of $50 \mathrm{sccm}$ was passed through the cell in order to drag the reaction products. A hydrogen flow was detected upon irradiation, showing a constant evolution and reaching an accumulated value of $2.35 \mu \mathrm{mol} / \mathrm{cm}^{2}$ after 60 minutes under irradiation (Figure $\mathbf{2 h}$ ), giving a constant increasing photocurrent (Figure S25). To check the stability of the system, a measurement of photocurrent at $0.2 \mathrm{~V}$ was conducted before and after $\mathrm{H}_{2}$ production, showing a consistent value (Figure S26).

Charge dynamics. To fully understand the photo(electro)catalytic behavior of IEF-5 it is key to elucidate the photoinduced charge transfer dynamics at different time scales, from fs to ms. For this purpose, we have combined core hole clock (CHC), time resolved photoluminescence (TRPL), and transient absorption spectroscopy (TAS) measurements.

Core hole clock (CHC) synchrotron experiments allow measuring charge delocalization rates in the attosecond scale for the first steps of charge separation. Such measurements can be performed on delocalized systems containing sulfur atoms ${ }^{45,46,47}$ by recording the S KLL Auger spectra at different excitation energies around the sulphur K-edge (Section S7). Figure 3 features a characteristic $2 \mathrm{D}$ plot of the CHC experiment for IEF-5 with three representative S KLL spectra. In the 2D spectrum (Figure 3a) it can be seen how the resonant signal increases in kinetic energy with excitation energy while the non-resonant signal shows always constant energy, as expected. Electron delocalization times are calculated from the S KLL Auger spectra using the life time of the $\mathrm{S} 1 \mathrm{~s}$ hole $(1.27 \mathrm{fs})^{48,49,50}$ and the area ratio between the non-resonant and resonant signals (Figure $\mathbf{c}$ and S27). Delocalization times are dependent on the conduction band orbital to which electrons are promoted varying from 0.2 
to 18 femtoseconds. These ultra-fast delocalization times are within the range of those calculated for a series of thiophene containing polymers. ${ }^{50}$ It can be seen how electron delocalization times decrease as the excitation energy increases, meaning that electrons promoted to more energetic conduction band orbitals (such as $\sigma^{*}$ or Rydberg states) exhibit faster delocalization than when they are promoted to lower lying energy states such as $\pi^{*}$.

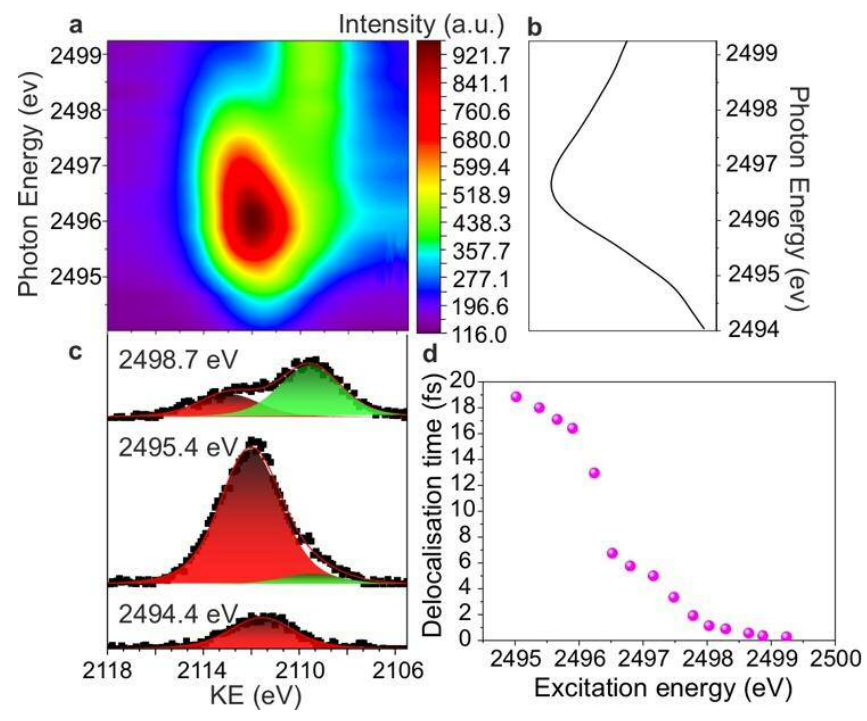

Figure 3. a) 2D Core hole clock measurements of IEF-5 including b) the NEXAFS scan (top right), c) representative S KLL spectra recorded at $2496.24 \mathrm{eV}, 2495.17 \mathrm{eV}$ and $2498.87 \mathrm{eV}$ excitation energies showing the resonant (red) and non-resonant (green) contributions to the Auger spectra and d) Electron delocalization times at the different excitation energies where the resonant peak is observed.

For a better understanding about charge photogeneration and transfer processes and their kinetic implications on the overall reaction pathways, a series of photophysical studies were performed. Solid state photoluminescence measurements show a significant quenching of the fluorescence emission in IEF-5, as compared to the linker (Figure 4a). Remarkably, time-resolved emission of IEF-5 (Figure 4a, inset) exhibits a singlet lifetime of $1.25 \mathrm{~ns}$, which is nearly 3 times higher than the corresponding to the free ligand $(0.45 \mathrm{~ns})$. In several studies, this behaviour is attributable to a ligand to metal charge transfer, through interaction between the carboxyl groups and bismuth atoms. ${ }^{51,52,53}$ However, taking into account TD-DFT calculation (see Section S4) the ligand to ligand charge transfer pathways seems more plausible.

Once the first steps in the light induced charge separation processes with IEF-5 have been depicted, we focused on investigating charge transfer kinetics, intermediate states, and their role in redox reactions. Considering that these processes occur in the $\mu$ s to ms timescale, laser flash photolysis (LPF) experiments were carried out. Transient absorption spectra (TAS) of IEF-5 exhibits a main band at $440 \mathrm{~nm}$, with a long lifetime over $25 \mu$ s showing a first-order kinetic (Figure S28). To unequivocally assign the observed transient, quenching experiments by oxygen were performed. ${ }^{54}$ It is well-known that the quenching of the triplet excited state of aromatic molecules by molecular oxygen $\left({ }^{3} \mathrm{O}_{2}\right)$ occurs via triplet energy transfer, ${ }^{55,56,57}$ being this process favored in long-lived excited triplet state. As expected for triplet, IEF-5 was efficiently quenched by oxygen with $K_{\mathrm{q}}=$ $3.6 \cdot 10^{8} \mathrm{M}^{-1} \mathrm{~s}^{-1}$ (Figure S29), confirming the triplet nature of the $440 \mathrm{~nm}$ transient. Moreover, the population dynamic between singlet and triplet states is heavily dependent of the nature of the media. In this context, besides the well-known effect of a heavy atom in the enhancement of the intersystem crossing rate (ISC), ${ }^{58,59,60,61}$ it has been reported the increase of ISC in small oligothiophenes by increasing the $\pi$-system compared to thiophene unit through calculation of spin-orbit coupling strength together with the singlet-triplet energy gap. ${ }^{62}$ In addition to that, recently it has been demonstrated that the presence of sulfur atoms and thiophene moieties in squaraine and arene rings, respectively, leads to significantly reducing the singlet-triplet energy difference, and in enhancement of the S1 $\left(\mathrm{n} \pi^{*}\right) \rightarrow \mathrm{T} 1\left(\pi \pi^{*}\right)$ transition. ${ }^{63,64}$ These reasons are consistent with the high triplet-triplet absorption population for IEF-5. On the other hand, the remarkable value monitored for triplet lifetime could be attributed to an efficient charge separation that can facilitate the $\mathrm{H}_{2}$ production reaction pathways. ${ }^{65,66}$

In addition, LPF experiments were performed in presence of electron-acceptor or electron-donor probe molecules to determine the charge transfer process, and intermediates involved in the reaction pathways. Measurements were first performed in presence of an electron donor such as $\mathrm{Na}_{2} \mathrm{SO}_{3}$ (Figure 4b), which we used as sacrificial agent in photo-electrochemical $\mathrm{H}_{2}$ production. The measurements exhibit a quenching in the 440 nm signal, attributed to the electron transfer from $\mathrm{SO}_{3}{ }^{2-}\left(\mathrm{E}^{0} \mathrm{SO}^{2-}\right.$ $/ \mathrm{SO}^{2-}=0.93 \mathrm{~V}$ vs NHE) ${ }^{67}$ to the MOF excited state.

More interestingly, a new transient band was observed at 515 $\mathrm{nm}$. This new species shows a huge increase in lifetime compared to the one corresponding to the $440 \mathrm{~nm}$ band (Figure 4c, inset). Moreover, a delay in the formation of IEF-5 signal was observed. This induction time can be attributed to diffusion processes limiting the charge transfer from the electron donor to IEF-5, and possibly this is the rate-determining step. This was further confirmed by observing how increasing amounts of sulfite anions lead to a progressive enhancement of the transient at $515 \mathrm{~nm}$ (Figure S30). The obtained transient was assigned to a MOF radical anion (IEF-5•-) due to the played role of sulfite as electron donor. In addition, it has been reported the formation of radicals from thiophene moieties in presence of electron donor / acceptor units. ${ }^{68}$ This assignment was confirmed by LPF measurements in presence of other electron donors, which have been employed in the literature to generate radical anions, ${ }^{69}$ such as triethylamine $\mathrm{Et}_{3} \mathrm{~N}(\mathrm{E}=0.96 \mathrm{~V}$ vs $\mathrm{SCE})$, triethanolamine TEOA or triethylenediamine DABCO $(\mathrm{E}(\mathrm{D} \cdot+\mathrm{D})=0.56$ $\mathrm{V}$ vs SCE) ${ }^{70}$ (Figure S31). Moreover, as expected due to the efficient triplet quenching by oxygen; under $\mathrm{O}_{2}$-saturated solutions, IEF-5•- radical anion signals were not detected, demonstrating that the transient observed at $515 \mathrm{~nm}$ is formed only from the excited states (Figure S32). Otherwise in presence of chloroplatinic acid (electron-acceptor) the signals attributable to triplet-triplet absorption were not observed (Figure 4c), indicating that photogenerated electrons are transferred to the acceptor to reduce platinum, in agreement with its potential as electron-acceptor $\left(\mathrm{E}_{[\mathrm{PtCl} 6] 2-/[\mathrm{PtCl} 4] 2-}^{0}=0.68 \mathrm{~V}, \mathrm{E}_{[\mathrm{PtCl} 4] 2-\mathrm{Pt}}^{0}=0.755\right.$ V). ${ }^{67,71}$ 

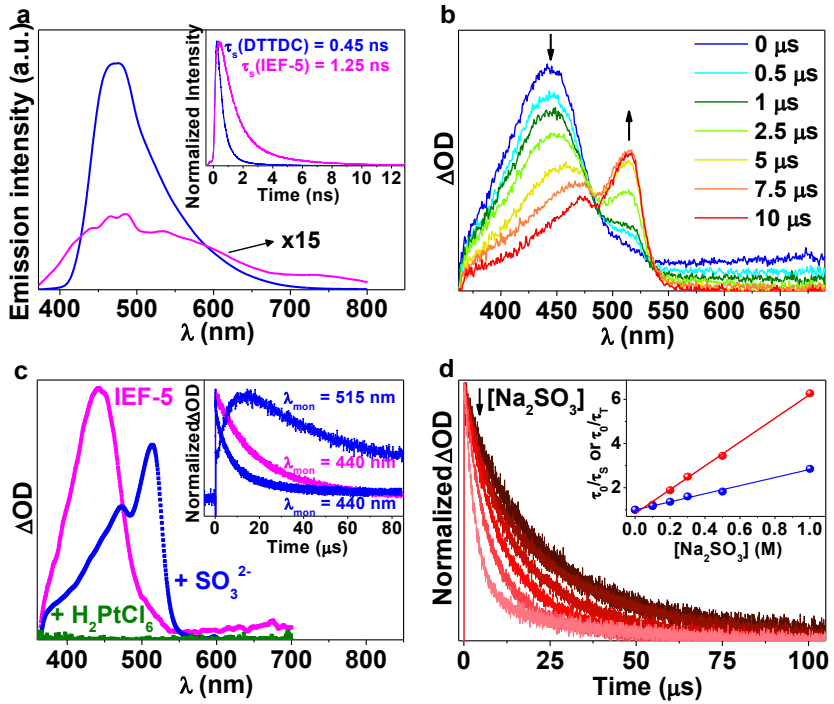

Figure 4. a) Emission spectra $\left(\lambda_{\text {exc }}=350 \mathrm{~nm}\right.$ ) of DTTDC (blue) and IEF-5 (magenta) in solid. Inset: Decay traces $\left(\lambda_{\text {exc }}=372 \mathrm{~nm}\right)$ of DTTDC and IEF-5; b) TAS obtained at different delay times $\left(\lambda_{\text {exc }}=355 \mathrm{~nm}\right)$ for IEF-5 in presence of $\mathrm{Na}_{2} \mathrm{SO}_{3} 0.5 \mathrm{M}$ as electron donor; c) TAS upon UV excitation $\left(\lambda_{\text {exc }}=355 \mathrm{~nm}\right.$ ) for IEF-5 (magenta) and using $\mathrm{H}_{2} \mathrm{PtCl}$ (green) as electron acceptor or $\mathrm{Na}_{2} \mathrm{SO}_{3}$ (blue) as electron donor. Inset: Triplet lifetimes monitored at 440 and $520 \mathrm{~nm}$ in absence (magenta) or in presence (blue) of $\mathrm{Nas}_{3}$; d) Changes in the triplet lifetimes $\left(\lambda_{\text {exc }}=355 \mathrm{~nm}\right)$ with increasing concentrations of $\mathrm{Na}_{2} \mathrm{SO}_{3}$. Insets: Stern-Volmer plot obtained from the kinetic analysis for singlet (blue) and triplet (red).

To corroborate the main contribution of triplet charge transfer pathway, quenching experiments using $\mathrm{Na}_{2} \mathrm{SO}_{3}$ were followed by time-resolved analysis monitoring singlet and triplet states. Stern-Volmer values $\left(k_{\mathrm{SV}}\right)$ obtained for IEF-5 were $K_{\mathrm{SV} \text { (S) }}=1.85$ and $K_{\mathrm{SV}(\mathrm{T})}=5.35 \mathrm{M}^{-1}$ for singlet and triplet measurements, respectively. The observed lower dynamic $k_{\mathrm{SV}}$ for singlet quenching (Figure S33) could be explained by the insignificant fluorescence quantum yield value of IEF-5. In fact, a low value of fluorescence quantum yield has been reported for the DTT mon$\operatorname{omer}\left(\phi_{\mathrm{F}}=0.01\right),{ }^{72}$ being probably much lower for IEF-5 in accordance with the results obtained through fluorescence measurements (Figure 4A). Thus, it can be assured that charge transfer from the electron donor occurs via excited triplet state. All these results demonstrate the key role of the MOF radical anion in the redox process, resulting in an improvement in the photocatalytic activity for IEF-5.

Based on the combination of experimental results, we can propose a reaction mechanism, which explains the involvement of charge-electron transfer processes in photocatalytic $\mathrm{H}_{2}$ production (Figure 5). After illumination IEF-5 suffers a charge separation, generating a singlet excited state $\left({ }^{1} \mathrm{IEF}-5^{*}\right)$ that undergoes a triplet excited state $\left({ }^{3} \mathrm{IEF}-5^{*}\right)$, by intersystem crossing (ISC). At this time, the hole scavenger $\left(\mathrm{SO}_{3}{ }^{2-}\right)$ interacts with the holes generated in IEF-5, injecting charge into the framework, with the subsequent formation of a radical anion intermediate state $\left(\right.$ IEF $\left.-5^{\circ}\right)$. This oxidation reaction can be performed on ${ }^{1}$ IEF-5* or ${ }^{3}$ IEF- $5 *$ states, being most probably the main contribution in the latter. On the other hand, photogenerated electrons in IEF-5 are transferred to the cathode $(\mathrm{Pt})$, where the redox cycle is closed by $\mathrm{H}_{2}$ evolution reaction.

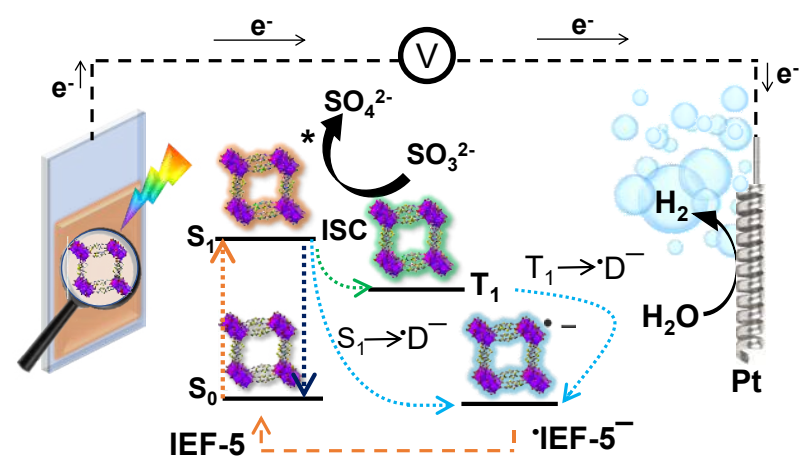

Figure 5. Scheme of photo(electro)catalytic $\mathrm{H}_{2}$ production mechanism, where $\mathrm{S}_{0}$ (Fundamental state), $\mathrm{S}_{1}$ (Singlet excited state); $\mathrm{T}_{1}$ (Triplet excited state), A (Anion) and ISC (intersystem crossing).

\section{CONCLUSIONS}

We have reported the synthesis of a novel bismuth-based MOF incorporating DTTDC as a hole transport linker specifically chosen for photoelectrocatalytic applications. This is among the few examples where bare MOFs are studied as photoanode under solar simulator irradiation for hydrogen production. Optoelectronic experimental and theoretical characterization indicates that the ligand plays the most important role in the charge transfer process, where the ligand to ligand transfer dominates over ligand to metal one.

Through the combination of CHC, TLF, and LPF, it has been possible to determine the intermediate states and to understand the charge dynamics behavior, which govern the kinetically controlled PEC reaction mechanism. These studies have shown that this involves a series of competitive processes in excited state at different time scales. First, charges are photogenerated and separated (fs) and transferred from excited state to triplet one (ns-ms) where the holes react with electron donors $\left(\mathrm{SO}_{3}{ }^{-}\right)$ through a radical anion intermediate. The electrons are transferred to the $\mathrm{Pt}$ cathode where $\mathrm{H}_{2}$ is produced. These results open new insights in the photo(electro)catalytic behavior of MOFs in light induced reactions for environmental remediation or solar fuel productions.

\section{ASSOCIATED CONTENT}

Supporting Information. Full experimental details. Crystallographic information file for IEF-5. This material is available free of charge via the Internet at http://pubs.acs.org.

\section{AUTHOR INFORMATION}

\section{Corresponding Author}

*gandara@icmm.csic.es

*victor.delapenya@imdea.org

\section{Author Contributions}

The manuscript was written through contributions of all authors. All authors have given approval to the final version of the manuscript.

\section{ACKNOWLEDGMENT}


This work was supported by the EU (ERC CoG HyMAP 648319) and Spanish MCIU, Ra-Phuel (ENE2016-79608-C21-R), SOLPAC (ENE2017-89170-R) FOTOFUEL (ENE201682025-REDT) and CTQ2017-87262-R (MCIU/AEI/FEDER, UE). We thank "Comunidad de Madrid" and European Structural Funds for their financial support to FotoArt-CM projec (S2018/NMT-4367). F.G., M. L and M. B thank to MINECO and European Social Fund for a Ramón y Cajal contract (RyC2015-18384, RyC-2015-18677) and Juan de la Cierva Formación contract (FJCI-2016-30567), respectively. We thank Diamond Facilities for the access to synchrotron radiation and CESCA for computational resources.

\section{RERERENCES}

(1) Blankenship, R. E.; Tiede, D. M.; Barber, J.; Brudvig, G. W.; Fleming, G.; Ghirardi, M.; Gunner, M. R.; Junge, W.; Kramer, D. M.; Melis, A.; et al. Comparing Photosynthetic and Photovoltaic Efficiencies and Recognizing the Potential for Improvement. Science 2011, 332 (6031), 805-809.

(2) Montoya, J. H.; Seitz, L. C.; Chakthranont, P.; Vojvodic, A.; Jaramillo, T. F.; Nørskov, J. K. Materials for Solar Fuels and Chemicals. Nat. Mater. 2016, 16, 70.

(3) $\mathrm{Su}$, J.; Vayssieres, L. A Place in the Sun for Artificial Photosynthesis? ACS Energy Lett. 2016, 1 (1), 121-135.

(4) de la Peña O'Shea, V. A.; Serrano, D. P.; Coronado, J. M. Current Challenges of $\mathrm{CO} 2$ Photocatalytic Reduction Over Semiconductors Using Sunlight BT - From Molecules to Materials: Pathways to Artificial Photosynthesis; Rozhkova, E. A., Ariga, K., Eds.; Springer International Publishing: Cham, 2015; pp 171-191. https://doi.org/10.1007/978-3-319-13800-8_7.

(5) Yang, W.; Prabhakar, R. R.; Tan, J.; Tilley, S. D.; Moon, J. Strategies for Enhancing the Photocurrent, Photovoltage, and Stability of Photoelectrodes for Photoelectrochemical Water Splitting. Chem. Soc. Rev. 2019, 48 (19), 4979-5015.

(6) Fresno, F.; Villar-García, I. J.; Collado, L.; AlfonsoGonzález, E.; Reñones, P.; Barawi, M.; de la Peña O’Shea, V. A. Mechanistic View of the Main Current Issues in Photocatalytic CO2 Reduction. J. Phys. Chem. Lett. 2018, 9 (24), 7192-7204.

(7) Fresno, F.; Portela, R.; Suárez, S.; Coronado, J. M. Photocatalytic Materials: Recent Achievements and near Future Trends. J. Mater. Chem. A 2014, 2 (9), 2863-2884.

(8) Jiang, C.; Moniz, S. J. A.; Wang, A.; Zhang, T.; Tang, J. Photoelectrochemical Devices for Solar Water Splitting - Materials and Challenges. Chem. Soc. Rev. 2017, 46 (15), 4645-4660.

(9) Sick, T.; Hufnagel, A. G.; Kampmann, J.; Kondofersky, I.; Calik, M.; Rotter, J. M.; Evans, A.; Döblinger, M.; Herbert, S.; Peters, K.; et al. Oriented Films of Conjugated 2D Covalent Organic Frameworks as Photocathodes for Water Splitting. J. Am. Chem. Soc. 2018, 140 (6), 2085-2092.

(10) Li, X.; Yu, J.; Gosztola, D. J.; Fry, H. C.; Deria, P. Wavelength-Dependent Energy and Charge Transfer in MOF: A Step toward Artificial Porous Light-Harvesting System. J. Am. Chem. Soc. 2019, 141 (42), 16849-16857.

(11) Deng, X.; Long, R.; Gao, C.; Xiong, Y. Metal-Organic Frameworks for Artificial Photosynthesis via Photoelectrochemical Route. Curr. Opin. Electrochem. 2019, 17, 114-120.

(12) Furukawa, H.; Cordova, K. E.; O'Keeffe, M.; Yaghi, O. M. The Chemistry and Applications of Metal-Organic Frameworks. Science. 2013, 341 (6149), 1230444.

(13) Dhakshinamoorthy, A.; Asiri, A. M.; García, H. MetalOrganic Framework (MOF) Compounds: Photocatalysts for Redox Reactions and Solar Fuel Production. Angew. Chemie Int. Ed. 2016, 55 (18), 5414-5445.

(14) Dolgopolova, E. A.; Rice, A. M.; Martin, C. R.; Shustova, N. B. Photochemistry and Photophysics of MOFs: Steps towards MOFBased Sensing Enhancements. Chem. Soc. Rev. 2018, 47 (13), 4710 4728.
(15) Xiao, J.-D.; Jiang, H.-L. Metal-Organic Frameworks for Photocatalysis and Photothermal Catalysis. Acc. Chem. Res. 2019, 52 (2), 356-366

(16) Butler, K. T.; Hendon, C. H.; Walsh, A. Electronic Chemical Potentials of Porous Metal-Organic Frameworks. J. Am. Chem. Soc. 2014, 136 (7), 2703-2706.

(17) Peng, Z.; Abbas, S. C.; Lv, J.; Yang, R.; Wu, M.; Wang, Y. Mixed-Metal Organic Framework-Coated ZnO Nanowires Array for Efficient Photoelectrochemical Water Oxidation. Int. J. Hydrogen Energy 2019, 44 (5), 2446-2453.

(18) Yoon, J. W.; Kim, D. H.; Kim, J. H.; Jang, H. W.; Lee, J. H. NH2-MIL-125(Ti)/TiO2 Nanorod Heterojunction Photoanodes for Efficient Photoelectrochemical Water Splitting. Appl. Catal. B Environ. 2019, 125, 511-518.

(19) Li, L.; Zhang, H.; Liu, C.; Liang, P.; Mitsuzaki, N.; Chen, Z. Effect of Co-Based Metal-Organic Framework Prepared by an In Situ Growth Method on the Photoelectrochemical Performance of Electrodeposited Hematite Photoanode. Energy Technol. 2019, 7 (5), 1801069

(20) Li, X.; Liu, S.; Fan, K.; Liu, Z.; Song, B.; Yu, J. MOF-Based Transparent Passivation Layer Modified ZnO Nanorod Arrays for Enhanced Photo-Electrochemical Water Splitting. Adv. Energy Mater. 2018, $8(18), 1-7$.

(21) Li, C. H.; Huang, C. L.; Chuah, X. F.; Senthil Raja, D.; Hsieh, C. T.; Lu, S. Y. Ti-MOF Derived Tix Fe1-xOy Shells Boost $\mathrm{Fe} 2 \mathrm{O} 3$ Nanorod Cores for Enhanced Photoelectrochemical Water Oxidation. Chem. Eng. J. 2019, 660-670.

(22) Xu, D.; Xia, T.; Fan, W.; Bai, H.; Ding, J.; Mao, B.; Shi, W. MOF-Derived Co3O4 Thin Film Decorated BiVO4 for Enhancement of Photoelectrochemical Water Splitting. Appl. Surf. Sci. 2019, 491, 497-504.

(23) Cardenas-Morcoso, D.; Ifraemov, R.; García-Tecedor, M.; Liberman, I.; Gimenez, S.; Hod, I. A Metal-Organic Framework Converted Catalyst That Boosts Photo-Electrochemical Water Splitting. J. Mater. Chem. A 2019, 7 (18), 11143-11149.

(24) Padial, N. M.; Castells-Gil, J.; Almora-Barrios, N.; RomeroAngel, M.; da Silva, I.; Barawi, M.; García-Sánchez, A.; de la Peña O'Shea, V. A.; Martí-Gastaldo, C. Hydroxamate Titanium-Organic Frameworks and the Effect of Siderophore-Type Linkers over Their Photocatalytic Activity. J. Am. Chem. Soc. 2019.

(25) Ifraemov, R.; Shimoni, R.; He, W.; Peng, G.; Hod, I. A Metal-Organic Framework Film with a Switchable Anodic and Cathodic Behaviour in a Photo-Electrochemical Cell. J. Mater. Chem. A 2019, 7 (7), 3046-3053.

(26) Kresse, G.; Furthmüller, J. Efficiency of Ab-Initio Total Energy Calculations for Metals and Semiconductors Using a PlaneWave Basis Set. Comput. Mater. Sci. 1996, 6 (1), 15-50.

(27) Kresse, G.; Hafner, J. Ab Initio Molecular Dynamics for Liquid Metals. Phys. Rev. B 1993, 47 (1), 558-561.

(28) Perdew, J. P.; Burke, K.; Ernzerhof, M. Generalized Gradient Approximation Made Simple. Phys. Rev. Lett. 1996, 77 (18), $3865-3868$

(29) Heyd, J.; Scuseria, G. E.; Ernzerhof, M. Hybrid Functionals Based on a Screened Coulomb Potential. J. Chem. Phys. 2003, 118 (18), 8207-8215.

(30) Momma, K.; Izumi, F. VESTA: A Three-Dimensional Visualization System for Electronic and Structural Analysis. J. Appl. Crystallogr. 2008, 41 (3), 653-658.

(31) Furche, F.; Ahlrichs, R. Adiabatic Time-Dependent Density Functional Methods for Excited State Properties. J. Chem. Phys. 2002, 117 (16), 7433-7447.

(32) Scalmani, G.; Frisch, M. J.; Mennucci, B.; Tomasi, J.; Cammi, R.; Barone, V. Geometries and Properties of Excited States in the Gas Phase and in Solution: Theory and Application of a TimeDependent Density Functional Theory Polarizable Continuum Model. J. Chem. Phys. 2006, 124 (9), 94107.

(33) Frisch, M. J.; Trucks, G. W.; Schlegel, H. B.; Scuseria, G. E.; Robb, M. A.; Cheeseman, J. R.; Scalmani, G.; Baro-ne, V.; Petersson, G. A.; Nakatsuji, H.; Li, X.; Caricato, M.; Marenich, A. V.; Bloino, J.; Janesko, B. G.; Gomperts, R.; Mennucci, B.; Hratc, D. J. Gaussian 16, Revision C.01. Gaussian Inc., Wallingford CT, 2016. 
(34) Becke, A. D. Density-Functional Exchange-Energy Approximation with Correct Asymptotic Behavior. Phys. Rev. A 1988, 38 (6), 3098-3100.

(35) Becke, A. D. Density-functional Thermochemistry. III. The Role of Exact Exchange. J. Chem. Phys. 1993, 98 (7), 5648-5652.

(36) Lee, C.; Yang, W.; Parr, R. G. Development of the ColleSalvetti Correlation-Energy Formula into a Functional of the Electron Density. Phys. Rev. B 1988, 37 (2), 785-789.

(37) Dunning, T. H.; Hay, P. J. Gaussian Basis Sets for Molecular Calculations BT - Methods of Electronic Structure Theory; Schaefer, H. F., Ed.; Springer US: Boston, MA, 1977; pp 1-27.

(38) Wadt, W. R.; Hay, P. J. Ab Initio Effective Core Potentials for Molecular Calculations. Potentials for Main Group Elements $\mathrm{Na}$ to Bi. J. Chem. Phys. 1985, 82 (1), 284-298.

(39) T. H. Dunning Jr. and P. J. Hay. Modern Theoretical Chemistry. In Modern Theoretical Chemistry; H. F. Schaefer III, V. 3, Ed.; Plenum, New York, 1977; pp 1-28.

(40) Strakova, K.; Assies, L.; Goujon, A.; Piazzolla, F.; Humeniuk, H. V; Matile, S. Dithienothiophenes at Work: Access to Mechanosensitive Fluorescent Probes, Chalcogen-Bonding Catalysis, and Beyond. Chem. Rev. 2019, 119 (19), 10977-11005.

(41) Wang, S.; Xiong, S.; Song, L.; Wang, Z. Influence of CoLigands and Solvents on the Packing and Photoluminescence of Three Related MnII Metal-Organic Frameworks. CrystEngComm 2009, 11 (5), 896-901.

(42) Wang, S.; He, X.; Song, L.; Wang, Z. Silver Nanoparticles Supported by Novel Nickel Metal-Organic Frameworks: An Efficient Heterogeneous Catalyst for an A3 Coupling Reaction. Synlett 2009, No. 3, 447-450.

(43) Bon, V.; Senkovskyy, V.; Senkovska, I.; Kaskel, S. Zr(IV) and Hf(IV) Based Metal-Organic Frameworks with Reo-Topology. 2012, 48 (67), 8407-8409.

(44) Briggs, D. Handbook of X-Ray Photoelectron Spectroscopy C. D. Wanger, W. M. Riggs, L. E. Davis, J. F. Moulder and G. E.Muilenberg Perkin-Elmer Corp., Physical Electronics Division, Eden Prairie, Minnesota, USA, 1979. Surf. Interface Anal. 1979, 3 (4).

(45) Arantes, C.; Borges, B. G. A. L.; Beck, B.; Roman, L. S.; Luiza, M.; Rocco, M. Femtosecond Electron Delocalization in Poly ( Thiophene ) Probed by Resonant Auger Spectroscopy Femtosecond Electron Delocalization in Poly ( Thiophene ) Probed by Ultrafast Electron Dynamics in the Low-Femtosecond Regime Was Evaluated For. J. Phys. Chem. 2013, No. 117, 8208-8213.

(46) Rocco, M. L. M.; Sekiguchi, T.; Baba, Y. Photon Stimulated Ion Desorption from Condensed Thiolane Photoexcited around the $\mathrm{S}$ 1s-Edge. J. Electron Spectros. Relat. Phenomena 2007, 156-158, 115118.

(47) Garcia-Basabe, Y.; Borges, B. G. A. L.; Silva, D. C.; Macedo, A. G.; Micaroni, L.; Roman, L. S.; Rocco, M. L. M. The Interplay of Electronic Structure, Molecular Orientation and Charge Transport in Organic Semiconductors: Poly(Thiophene) and Poly(Bithiophene). Org. Electron. physics, Mater. Appl. 2013, 14 (11), 2980-2986.

(48) Rocco, M. L. M.; Sekiguchi, T.; Baba, Y. Photon Stimulated Ion Desorption from Condensed Thiophene Photoexcited Around the S1s-Edge. J. Vac. Sci. Technol. A 2006, 24 (6), 2117-2121.

(49) Hitchcock, A. P.; Horsley, J. A.; Stöhr, J. Inner Shell Excitation of Thiophene and Thiolane: Gas, Solid, and Monolayer States. J. Chem. Phys. 1986, 85 (9), 4835-4848.

(50) Garcia-basabe, Y.; Borges, B. G. A. L.; Silva, D. C.; Macedo, A. G.; Micaroni, L.; Roman, L. S.; Rocco, M. L. M. The Interplay of Electronic Structure, Molecular Orientation and Charge Transport in Organic Semiconductors: Poly (Thiophene) and Poly (Bithiophene). Org. Electron. 2013, 14 (11), 2980-2986.

(51) Wuttke, S.; Dietl, C.; Hinterholzinger, F. M.; Hintz, H.; Langhals, H.; Bein, T. Turn-on Fluorescence Triggered by Selective Internal Dye Replacement in MOFs. Chem. Commun. 2014, 50 (27), 3599-3601.

(52) Keller, N.; Bessinger, D.; Reuter, S.; Calik, M.; Ascherl, L.; Hanusch, F. C.; Auras, F.; Bein, T. Oligothiophene-Bridged Conjugated Covalent Organic Frameworks. J. Am. Chem. Soc. 2017, 139 (24), 8194-8199.
(53) Wang, G.; Sun, Q.; Liu, Y.; Huang, B.; Dai, Y.; Zhang, X.; Qin, X. A Bismuth-Based Metal-Organic Framework as an Efficient Visible-Light-Driven Photocatalyst. Chem. - A Eur. J. 2014, 20, 1-5.

(54) Paris, C.; Lhiaubet-Vallet, V.; Jiménez, O.; Trullas, C.; Miranda, M. Á. A Blocked Diketo Form of Avobenzone: Photostability, Photosensitizing Properties and Triplet Quenching by a Triazine-Derived UVB-Filter. Photochem. Photobiol. 2009, 85 (1), $178-184$.

(55) Patterson, L. K.; Porter, G.; Topp, M. R. Oxygen Quenching of Singlet and Triplet States. Chem. Phys. Lett. 1970, 7 (6), 612-614.

(56) Grewer, C.; Brauer, H.-D. Mechanism of the Triplet-State Quenching by Molecular Oxygen in Solution. J. Phys. Chem. 1994, 98 (16), 4230-4235.

(57) Abdel-Shafi, A. A.; Worrall, D. R. Mechanism of the Excited Singlet and Triplet States Quenching by Molecular Oxygen in Acetonitrile. J. Photochem. Photobiol. A Chem. 2005, 172 (2), 170 179.

(58) Filatov, M. A. Heavy-Atom-Free BODIPY Photosensitizers with Intersystem Crossing Mediated by Intramolecular Photoinduced Electron Transfer. Org. Biomol. Chem. 2019.

(59) Cui, G.; Fang, W. State-Specific Heavy-Atom Effect on Intersystem Crossing Processes in 2-Thiothymine: A Potential Photodynamic Therapy Photosensitizer. J. Chem. Phys. 2013, 138 (4), 44315.

(60) Barradas, I.; Ferreira, J. A.; Thomaz, M. F. Intramolecular Heavy-Atom Effect and Intersystem-Crossing in Monohalogenated Pyrenes. J. Chem. Soc. Faraday Trans. 2 Mol. Chem. Phys. 1973, 69 (0), 388-394.

(61) Yoon, J.; Jung, Y. J.; Yoon, J. B.; Damodar, K.; Kim, H.; Shin, M.; Seo, M.; Cho, D. W.; Lee, J. T.; Lee, J. K. The Heavy-Atom Effect on Xanthene Dyes for Photopolymerization by Visible Light. Polym. Chem. 2019, 10 (42), 5737-5742.

(62) Kölle, P.; Schnappinger, T.; de Vivie-Riedle, R. Deactivation Pathways of Thiophene and Oligothiophenes: Internal Conversion versus Intersystem Crossing. Phys. Chem. Chem. Phys. 2016, 18 (11), 7903-7915.

(63) Sadiq, F.; Zhao, J.; Hussain, M.; Wang, Z. Effect of Thiophene Substitution on the Intersystem Crossing of Arene Photosensitizers. Photochem. Photobiol. Sci. 2018, 17 (11), 1794 1803.

(64) Peceli, D.; Hu, H.; Fishman, D. A.; Webster, S.; Przhonska, O. V; Kurdyukov, V. V; Slominsky, Y. L.; Tolmachev, A. I.; Kachkovski, A. D.; Gerasov, A. O.; et al. Enhanced Intersystem Crossing Rate in Polymethine-Like Molecules: Sulfur-Containing Squaraines versus Oxygen-Containing Analogues. J. Phys. Chem. A 2013, 117 (11), 2333-2346.

(65) Collado, L.; Reynal, A.; Fresno, F.; Barawi, M.; Escudero, C.; Perez-Dieste, V.; Coronado, J. M.; Serrano, D. P.; Durrant, J. R.; de la Peña O'Shea, V. A. Unravelling the Effect of Charge Dynamics at the Plasmonic Metal/Semiconductor Interface for $\mathrm{CO} 2$ Photoreduction. Nat. Commun. 2018, 9 (1), 4986.

(66) de Miguel, M.; Ragon, F.; Devic, T.; Serre, C.; Horcajada, P.; García, H. Evidence of Photoinduced Charge Separation in the Metal-Organic Framework MIL-125(Ti)-NH2. ChemPhysChem 2012, 13 (16), 3651-3654.

(67) Vanýsek, P. Electrochemical Series (Standard Reduction Potential Tables). CRC Handb. Chem. Physics, 91th Ed. 2010, No. 2, $5-86$.

(68) Fujitsuka, M.; Sato, T.; Segawa, H.; Shimidzu, T. Photochemical Polymerization of Oligothiophene and Dithienothiophene. Synth. Met. 1995, 69 (1), 309-310.

(69) Martinez-Haya, R.; Miranda, M. A.; Marin, M. L. MetalFree Photocatalytic Reductive Dehalogenation Using Visible-Light: A Time-Resolved Mechanistic Study. European J. Org. Chem. 2017, 2017 (15), 2164-2169.

(70) S. L. Murov, I. Carmichael, G. L. H. Handbook of Photochemistry, 2nd ed.; Dekker, M., Ed.; New York, 2009.

(71) Bard Allen J.; Faulkner Larry R. Electrochemical Methods: Fundamentals and Applications, 2nd Editio.; John Wiley and Sons Inc., 2001. 
(72) Wang, C.-Z.; Do, J.-H.; Akther, T.; Feng, X.; Horsburgh, L.; Elsegood, M. R. J.; Redshaw, C.; Yamato, T. D- $\pi$-D Chromophores

Based on Dithieno[3,2-b:2',3'-d]Thiophene (DTT): Potential
Application in the Fabrication of Solar Cell. Tetrahedron 2017, 73 (4), 307-312. 